\title{
Treatment Options in the Management of Status Epilepticus
}

\section{Andrea 0. Rossetti}

\author{
Address \\ Service de Neurologie, CHUV-BH07 CH-1011, Lausanne, Switzerland \\ Email: andrea.rossetti@chuv.ch \\ Published online: 26 February 2010 \\ (C) Springer Science+Business Media, LLC 2010
}

\section{Opinion statement}

Status epilepticus treatment involves the use of several pharmacologic compounds, which are conceptually divided into three successive and additional lines of action. Because of their rapid onset of action, benzodiazepines represent the first approach; these are followed by classic antiepileptic drugs that are administered intravenously. In refractory episodes, pharmacologic coma induction with an appropriate anesthetic is advocated. Apart from first-line compounds, the level of evidence for medications used in status epilepticus is extremely limited. It is important to specifically address etiology in order to maximize the impact of the antiepileptic therapy. Fine-tuning of the treatment strategy, mainly regarding the choice of whether to induce coma, should be approached by balancing the benefits of rapid control of the status epilepticus with the risks of adverse effects. Although each status epilepticus episode should be treated as rapidly as possible, it appears advisable to reserve coma induction for those forms, such as generalized convulsive status, that have been shown to present a consistent risk of neurologic sequelae.

\section{Introduction}

Status epilepticus (SE), the most common neurologic emergency after stroke, requires multidisciplinary management, including paramedics and nurses, neurologists, emergency specialists, and intensive care unit physicians; at times, pharmacologists and members of other medical specialties (eg, infectious disease specialists or immunologists) complete the team. SE must be treated as soon as possible in order to prevent potentially harmful complications such as functional neurologic and neuropsychological impairment or even death [1-3]. Despite the important impact of $\mathrm{SE}$, to date there is only a relatively low degree of evidence concerning its pharmacologic treatment, perhaps because of the marked variability of SE forms and the challenges of designing large collaborative trials (including the difficulty of gathering financial support). As a result, past therapy strategies have been relatively heterogeneous. Although in recent years several national and international guidelines have been published, it remains unclear whether these are thoroughly applied and whether they have led to an improvement of SE prognosis. Moreover, it seems important to adapt treatments to the specific circumstances of each patient, in 
order to maximize the ratio between efficacy and adverse effects.

This paper reviews pharmacologic agents used in SE treatment and gives some practical suggestions about treatment options and strategies.

\section{General treatment considerations}

Seizures (and, if they are prolonged, SE) result from an imbalance between inhibitory neuronal inputs (mostly through $\mathrm{GABA}_{\mathrm{A}}$ receptors) and excitatory inputs (predominantly glutamate-mediated, via kainate and AMPA receptors) $[1,4]$. This characteristic may be viewed as a rationale for beginning SE treatment with benzodiazepines, which are rapidly acting GABAergic agents. However, in ongoing SE, GABA resistance develops progressively, following receptor internalization into the cell and subunit changes; this process leads to a shift toward self-sustaining glutamate-mediated excitotoxicity, resulting primarily from the activation of NMDA receptors. These changes likely explain both refractoriness to benzodiazepines and excitotoxic neuronal damage.

SE treatment aims at stopping seizure activity and concomitantly preventing complications. First, pulmonary and cardiac functions need to be secured; in parallel, a targeted examination and history-taking must be performed to detect possible SE imitators, such as movement disorders (eg, shivering in the ICU) or psychogenic seizures, which may account for a not-negligible proportion of "convulsions" in the emergency room [5]. Sequential laboratory and neuroradiologic workups are needed to address SE etiology, which may greatly influence the success of specific treatments, including the choice of antiepileptic drugs (AEDs). As a rule of thumb, SE treatment should be performed with the help of electroencephalography (EEG). Prolonged recordings are best, as they not only allow judgments about the efficacy of administered agents but also are required for the diagnosis of nonconvulsive SE (ie, complex-partial SE, absence SE, or SE in coma).

\section{Treatment}

- It is useful to categorize pharmacologic SE treatment into three steps [2], bearing in mind that, in general, intravenous administration is to be preferred because of the possibility of fast loading. The first treatment line is represented exclusively by benzodiazepines aiming at rapid SE control, the second line by classic AEDs targeting early resistant forms of SE and long-term coverage following anticipated control of SE, and the third line by general anesthetics used for refractory SE. This approach should be not only sequential, meaning that the next step is initiated as soon as the previous one is considered to have failed, but also additional, implying that previous treatment lines should not be discontinued, thus allowing complementary actions thanks to different pharmacodynamic mechanisms. A simple protocol with corresponding timing is proposed in Fig. 1. Awareness of a protocol allows a smooth interplay between different team members (paramedics, emergency or ICU team, neurologists) and probably reduces wasted time during emergency management. However, every protocol, even if simple, should be interpreted according to the situation and modified as needed. Of note, if a glucose solution is administered to the patient, it is important to add thiamine to prevent a Wernicke encephalopathy.

- Although multiple pharmacologic options are used to treat SE, there is a substantial lack of comparative, high-level, evidence-based information. Use of a treatment protocol with the three steps is recom- 


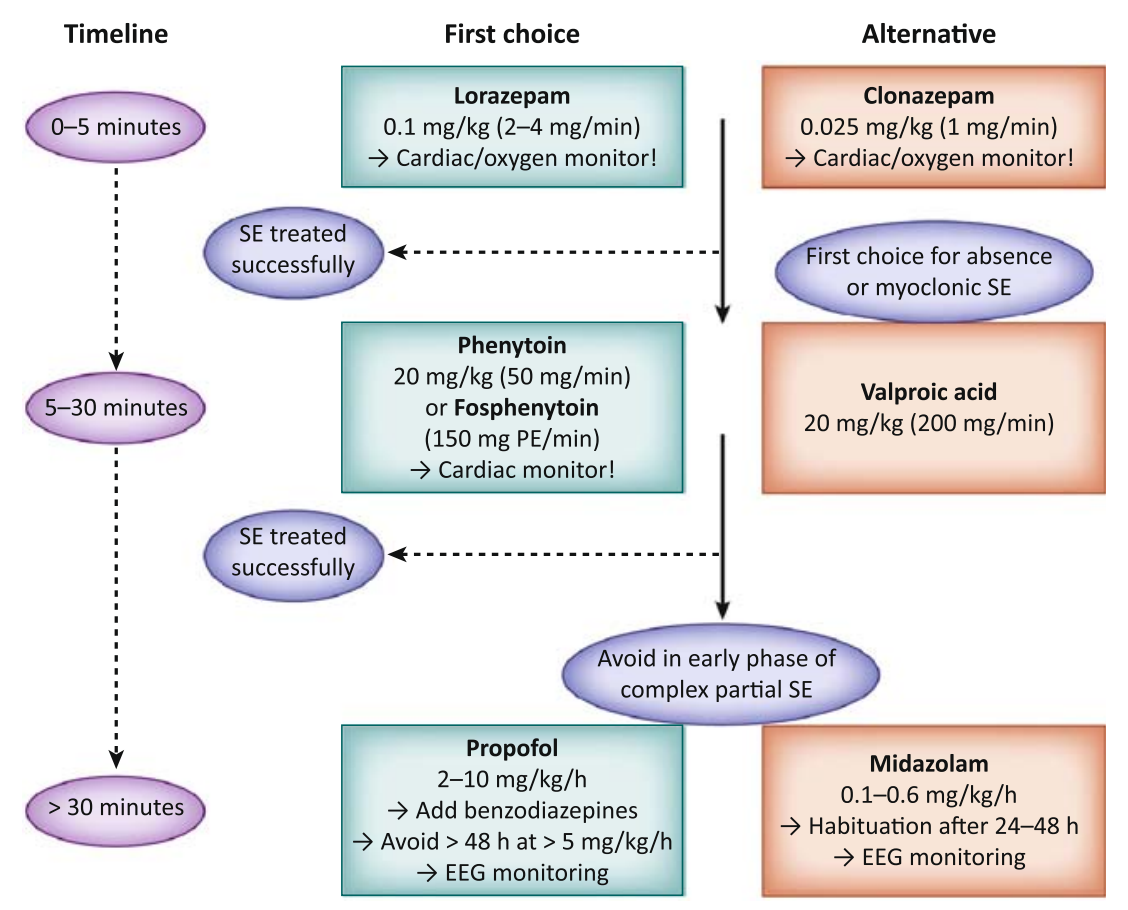

Figure 1. Overview of pharmacologic treatment of status epilepticus (SE). This protocol is by no means highly evidencebased, but only summarizes the author's opinion and experience. EEGelectroencephalography.

mended in order to facilitate and accelerate the early practical management. The patient's background, including etiology and age, largely determines the prognosis, so it is important to consider these aspects and to adapt the treatment strategy to each particular case, in order to minimize the risks of overtreatment or undertreatment. The art of treating SE effectively resides more in the fine-tuning of the approach than in the automatic application of sequential agents. It is hoped that future trials will draw attention to the best strategy for the management of this heterogeneous condition.

\section{First treatment line}

- This is the only treatment step that has been studied in several randomized controlled trials. The first, carried out more than 20 years ago on a relatively limited number of patients, disclosed a nonsignificant trend toward better response to $4 \mathrm{mg}$ lorazepam as compared to $10 \mathrm{mg}$ diazepam [6, Class I]. Similarly, a large prehospital trial at the beginning of this decade, using relatively low doses, found that $2 \mathrm{mg}$ lorazepam had a nonsignificant superiority over $5 \mathrm{mg}$ diazepam, both treatments were significantly better than placebo, and cardiovascular and respiratory complications were similar among all three groups [7, Class I], suggesting that SE per se may be responsible for causing hypotension and respiratory depression. A large VA trial, focusing on generalized convulsive SE and assessing the efficacy of $0.1 \mathrm{mg} / \mathrm{kg}$ lorazepam, $15 \mathrm{mg} / \mathrm{kg}$ phenobarbital, $0.15 \mathrm{mg} / \mathrm{kg}$ diazepam followed by $18 \mathrm{mg} / \mathrm{kg}$ phenytoin, and $18 \mathrm{mg} / \mathrm{kg}$ phenytoin 


\begin{tabular}{|c|c|c|}
\hline & Standard dosage & $\begin{array}{l}\text { Lorazepam is administered as a slow bolus of } 0.1 \mathrm{mg} / \mathrm{kg}(2 \mathrm{mg} / \mathrm{min}) \text { it enters } \\
\text { the brain in less than } 2 \text { to } 3 \text { minutes }[10] \text { and has a long duration of action } \\
\text { (at least } 12 \mathrm{~h}) \text {, because it is less likely than diazepam to redistribute in the fat } \\
\text { tissue }[11] \text {; the protein binding is estimated at } 90 \% \text {. Its elimination half-life } \\
\text { is about } 8 \text { to } 25 \mathrm{~h}[10,11]\end{array}$ \\
\hline & Contraindications & Hypersensitivity to the compound; absence of respiratory monitoring. \\
\hline & Main drug interactions & None. \\
\hline & Main side effects & Sedation, respiratory depression. \\
\hline \multicolumn{3}{|l|}{ Clonazepam } \\
\hline & Standard dosage & $\begin{array}{l}\text { Clonazepam is administered at a bolus of } 0.025 \mathrm{mg} / \mathrm{kg} \text {. It reaches the brain } \\
\text { within } 1 \text { minute [10], and, despite its lipophilia, has a stable action over } \\
\text { time. It has a plasma half-life of up to } 38 \mathrm{~h} \text { and moderate protein binding } \\
(65 \%) \text { [12]. It is relatively widely used in Europe, but it is not approved by } \\
\text { the US Food and Drug Administration as a treatment for SE. }\end{array}$ \\
\hline & Contraindications & Hypersensitivity to the compound; absence of respiratory monitoring. \\
\hline & Main drug interactions & None. \\
\hline & Main side effects & Sedation, respiratory depression. \\
\hline \multicolumn{3}{|l|}{ Midazolam } \\
\hline & Standard dosage & $\begin{array}{l}\text { Midazolam usually is given at a dose of } 0.1 \text { to } 0.2 \mathrm{mg} / \mathrm{kg} \text {, but doses up to } \\
0.5 \mathrm{mg} / \mathrm{kg} \text { have been reported [13]. It has a short half-life (about } 2 \mathrm{~h} \text { ) but } \\
\text { represents a valuable alternative when intravenous lines are not available } \\
\text { or in treating children, as intranasal or buccal administration is possible. } \\
\text { Protein binding is very high }(97 \%) \text {. }\end{array}$ \\
\hline & Contraindications & Hypersensitivity to the compound; absence of respiratory monitoring. \\
\hline
\end{tabular}

alone, disclosed better efficacy of lorazepam versus phenytoin alone, but not in comparison to the other arms [8, Class I]. Of note, this was the only trial adapting the dosage to body weight. The overall response in overt SE (about 60\%) was higher than in subtle SE (about 20\%).

- SE becomes more refractory to treatment with time, and the first treatment has a far better chance of success than the second or third, regardless of the drug ( $55 \%$ vs $7 \%$ vs $2 \%$ in the VA trial) [9, Class I], so it appears mandatory to administer intravenous drugs that act quickly. In this view, benzodiazepines are the best alternative, over phenobarbital and phenytoin. Compounds with a long elimination half-life in the central nervous system (CNS) are preferred, to minimize the risk of withdrawal or rebound seizures as drug levels decline.

- The administration of a benzodiazepine bolus during SE may induce respiratory and circulatory collapse (about $10 \%$ to $26 \%$ ) [7,8]; therefore, monitoring of these functions is mandatory during administration of benzodiazepines.

- Note that tonic SE in patients with developmental delay, suffering from epileptic encephalopathies, may rarely be aggravated by benzodiazepines. 
Main drug interactions None.

Main side effects Sedation, respiratory depression.

Diazepam

Standard dosage Diazepam is administered intravenously at $0.2 \mathrm{mg} / \mathrm{kg}(5 \mathrm{mg} / \mathrm{min})$; it enters the brain in less than $10 \mathrm{~s}$, but its free fraction redistributes in fat tissue owing to its high lipophilia. Therefore, after about 20 minutes, drug levels in the brain decline significantly [11]. It is tightly bound to proteins (99\%). This agent also may be administered rectally—a helpful alternative, especially for children.

Contraindications Hypersensitivity to the compound; absence of respiratory monitoring.

Main drug interactions

None.

Main side effects Sedation, respiratory depression.

\section{Second treatment line}

Phenytoin

- No large-scale, prospective comparative assessments among AEDs used as second-line or third-line SE treatment have been performed to date. The VA study included an arm using phenytoin (which acts principally through sodium channel modulation) as initial SE treatment and an arm using phenobarbital (mainly a $\mathrm{GABA}_{\mathrm{A}}$ agonist); it found a nonsignificant trend toward better efficacy with phenobarbital (58\% vs $44 \%$ [8]).

- Intravenous valproate has been repeatedly reported to be efficacious for several SE types $[14,15]$, probably in view of its multimodal pharmacodynamic action, including GABA, sodium channel, and calcium channel modulations. It lacks significant cardiovascular adverse reactions, so there is no need of concurrent monitoring, a potential advantage in out-of-hospital situations. Some studies comparing it with phenytoin were recently published [14,16,17; Class III], but they are relatively small and suffer from major methodologic pitfalls.

- Levetiracetam has also been employed in SE treatment for several years [18]; the recent availability of an intravenous form renders it even more promising [19•,20•; Class IV]. It has good tolerability, but no comparative trial has been published to date.

- The recently marketed lacosamide was available in an intravenous form from the beginning; it acts by modulating the slow inactivation of the sodium channel and by interfering with the collapsin protein function. Experience in SE is still anecdotal, however [21•, Class IV].

- After loading of one of these agents, serum levels should be checked periodically to ensure therapeutic concentrations.

Standard dosage

Phenytoin is loaded at $20 \mathrm{mg} / \mathrm{kg}$ (maximal infusion rate, $50 \mathrm{mg} / \mathrm{min}$ ). Maximal concentrations in the CNS are reached after 20 minutes [11]. The elimination half-life is about $24 \mathrm{~h}$, but it becomes significantly longer at high serum levels. A slower infusion rate is especially advisable for elderly patients, and cardiac monitoring should always be performed during intra- 
Contraindications

Main drug interactions

Main side effects

Fosphenytoin venous administration. Phenytoin is probably the most frequently used second-line agent.

Complex cardiac arrhythmias, absence of cardiac and respiratory monitoring. Phenytoin is a potent enzyme inductor.

Some rare but serious local reactions (eg, purple glove syndrome) are induced by the alkaline solution, whereas phenytoin itself was associated with hypotension (27\%) and bradyarrhythmia (7\%) in the VA study group [8]).
Standard dosage

Contraindications

Main drug interactions

Main side effects

Special points

Phenobarbital
Fosphenytoin is a water-soluble phenytoin prodrug that lacks propylene glycol and therefore is safer as regards local tissue reactions. It is dosed in phenytoin equivalents (PE). Although it can be infused at a faster rate than phenytoin (150 mg PE/min), it is questionable whether effective CNS concentrations are reached more quickly than when phenytoin is administered at optimal rates [22].

Same as phenytoin.

Same as phenytoin.

Hypotension and bradyarrhythmia.

Cost limits its availability in some countries.
Standard dosage

Contraindications

Main drug interactions

Main side effects

Special points

Valproic acid
Phenobarbital is loaded at $15 \mathrm{mg} / \mathrm{kg}(100 \mathrm{mg} / \mathrm{min})$. It reaches the brain after 20 to 40 minutes and has a long half-life of about $100 \mathrm{~h}$.

Absence of cardiac and respiratory monitoring.

Phenobarbital is a potent enzyme inductor.

Phenobarbital also bears a consistent risk of hypotension (34\% in the VA study [8]).

The use of phenobarbital as a first-choice second-line agent is relatively rare.

Standard dosage Valproic acid is loaded at $20 \mathrm{mg} / \mathrm{kg}$, at high rates (up to $200 \mathrm{mg} / \mathrm{min}[14,23]$ ) its elimination half-life is about $15 \mathrm{~h}$. Valproic acid enters the CNS rapidly through active transport [24]. Clinical experience in SE suggests that effective CNS concentrations are reached within 30 minutes [23,25]. Its main advantage is the lack of cardiodepressive reactions.

Contraindications Active hepatitis, pancreatitis, mitochondrial disorder.

Main drug interactions Valproic acid is an enzyme inhibitor.

Main side effects At times, follow-up of ammonium counts (especially in patients with persisting slow EEGs) may be advisable, in order to detect an hyperammonemic encephalopathy.

Special points Valproic acid is increasingly used for SE, especially in settings devoid of cardiac and respiratory monitoring. Moreover, it represents the first choice for absence or myoclonic SE. 
Contraindications

Main drug interactions

Main side effects

Special points longer [26]. It is still unclear how quickly levetiracetam reaches the brain. Monitoring of platelet counts may be advisable [19•].

Hypersensitivity to the compound.

None.

The most frequent adverse event is mild sedation; no cardiovascular reactions have been reported.

More expensive than other second-line agents.

Lacosamide

Standard dosage

Contraindications

Main drug interactions

Main side effects

Special points
Loading doses of up to $400 \mathrm{mg}$ have been described [21•].

Hypersensitivity to the compound.

None known.

Mild sedation.

More expensive than other second-line agents; only anecdotal reports to date.

\section{Third treatment line}

\section{Barbiturates}

- In generalized convulsive SE, the first treatment administered is the most likely to be effective $[9,27]$. For this reason, it seems reasonable to proceed straight to third-line treatment once the second-line (which takes at least 20 to 30 minutes to be effective) has failed $[2,28 \bullet \bullet]$. The available studies on refractory SE consist of more or less large case series. A meta-analysis comparing barbiturates with propofol (both acting mainly through modulation of $\mathrm{GABA}_{\mathrm{A}}$ receptors) and midazolam did not show any significant difference in short-term mortality with these three agents, although some variations were noted in efficacy (somewhat better for barbiturates) and tolerability (somewhat worse for barbiturates); the patients treated with barbiturates were more likely to be monitored with EEG, however, which may explain some of the differences [29, Class IV]. A retrospective analysis taking into account possible anesthetic combinations did not disclose any notable outcome difference among the agents, whether used alone or in association [30, Class IV].

- There is also considerable uncertainty about the optimal extent of pharmacologic EEG suppression [30] and treatment duration. An EEG target of burst suppression with an interburst interval of about $10 \mathrm{~s}$, maintained for 24 to $36 \mathrm{~h}$ and followed by progressive tapering over 12 to $48 \mathrm{~h}$, represents a good, practical option, but this regimen is not yet supported by any comparative assessment.

These agents include thiopental in Europe or its metabolite pentobarbital in North America.

Standard dosage Induction with pentobarbital is performed with boluses of 5 to $15 \mathrm{mg} / \mathrm{kg}$ the maintenance dose is 1 to $5 \mathrm{mg} / \mathrm{kg}$ per hour. Thiopental is loaded at repetitive boluses of $2 \mathrm{mg} / \mathrm{kg}$ and then relayed with a drip of 3 to $5 \mathrm{mg} / \mathrm{kg}$ per hour. 
Contraindications Need for short-term mechanical ventilation (eg, patients with chronic obstructive pulmonary disease).

Main drug interactions Barbiturates are potent enzyme inductors.

Main side effects Hypotension and respiratory depression.

Standard dosage

Contraindications

Main drug interactions

Main side effects
These agents show a long half-life after continuous administration (pentobarbital, 15h22h; thiopental, probably slightly longer) [31], and they have a considerable tendency to accumulate in fat tissue, prolonging the need for mechanical ventilation.

The loading dose is $2 \mathrm{mg} / \mathrm{kg}$, followed by maintenance at 2 to $10 \mathrm{mg} / \mathrm{kg}$ per hour. Propofol has a short half-life (about 1-2 h [32]), allowing rapid titration and withdrawal.

Metabolic acidosis, hypertriglyceridemia, mitochondrial disorder.

None.

Sedation and respiratory depression. Especially in young children, it may induce the "propofol infusion syndrome" (PRIS), a potentially fatal cardiocirculatory collapse characterized by lactic acidosis, hypertriglyceridemia, rhabdomyolysis, and green-colored urine; PRIS has been rarely described in patients with SE $[33,34 \bullet]$. Concomitant benzodiazepine administration (eg, a clonazepam or midazolam drip or repetitive short infusions of lorazepam) could lower the propofol dose needed to obtain seizure control, possibly reducing the risk of this complication [35]. Administration of doses over $5 \mathrm{mg} / \mathrm{kg}$ per hour for longer than $48 \mathrm{~h}$ should be avoided, and repetitive checks of serum lactate could help to detect a beginning PRIS in time to discontinue propofol.

Standard dosage

Contraindications

Main drug interactions

Main side effects

Special points
Midazolam is loaded at $0.2 \mathrm{mg} / \mathrm{kg}$, and then maintained at 0.1 to $0.6 \mathrm{mg} / \mathrm{kg}$ per hour. It has a half-life of 6 to $40 \mathrm{~h}$ after prolonged infusion [36], with an important habituation (tachyphylaxis) developing within 24 to $48 \mathrm{~h} \mathrm{[37].}$

Hypersensitivity to the compound.

None.

Sedation and respiratory depression.

In view of the tachyphylaxis, which requires large dosage increases after the first day, it may be advisable to switch to one of the other options if prolonged treatment becomes necessary.

\section{Other treatment approaches for persistent, refractory SE}

- When SE proves resistant to the first three lines of treatment, usually several days have elapsed since the beginning of the episode. At this moment, it is advisable to attempt switching to another anesthetic of the third line; doing this gives some time to titrate one further secondline compound. It is paramount not to forget to periodically address SE 
etiology, because SE prognosis appears to be related mainly to etiology rather than to treatment approach $[30,38 \bullet, 39 \bullet]$. Some cases of SE prove extremely refractory, however; this condition has been labeled "malignant" and is encountered somewhat more often in young patients with some form of inflammatory disease [40]. Other treatment strategies, used relatively infrequently, have been described in anecdotal case reports or small series; these may be employed in succession. It is unlikely that these strategies will influence the outcome in a decisive way, but some may be interesting in particular settings. Of course, safety should always remain a priority for the prescribing physician.

- Other anesthetics, such as ketamine, which acts as an NMDA antagonist $[41 \bullet$, Class IV], or isoflurane [42, Class IV], an inhalation compound, could be worth a trial. Ketamine may represent an interesting option in view of the pathophysiology of SE; it should be combined with benzodiazepines in order to minimize the risk of neuronal toxicity.

- High-dose oral topiramate, administered through the nasogastric tube, could be beneficial at times [43, Class IV]; this agent may modulate AMPA receptors, in addition to sodium and calcium channels.

- Verapamil, a calcium channel blocker, inhibits the P-glycoprotein, a multidrug transporter diminishing AED availability in the brain. Few case reports on its use in humans with SE are available. It appears relatively safe (under cardiac monitoring) up to dosages of $360 \mathrm{mg} / \mathrm{d}$ [44, Class IV].

- The ketogenic diet has been prescribed for decades, mostly for children, to control refractory seizures; its use in refractory SE as a last resort has been described occasionally [45, Class IV]. Its effect is exerted over several days to a few weeks.

- Immunomodulatory approaches such as steroids, intravenous immunoglobulin, or plasma exchanges are often tried in patients with very refractory SE [46, Class IV], at times even without evidence of an underlying inflammatory etiology.

- Potential nonpharmacologic treatments include vagus nerve stimulation initiated in the operating room [47, Class IV], mild transitory hypothermia [48•, Class IV], and resective surgery of an isolated epileptic focus distant from eloquent areas [49, Class IV].

It is my opinion that an important exception to the rule of treating SE quickly and aggressively concerns SE episodes (including absence SE and several forms of complex partial SE) in which patients are at least partly conscious. Indeed, absence SE nearly always resolves without sequelae, and it is still undetermined whether prolonged complex partial seizures in humans induce 
permanent structural neurologic damage [50,51]. This differs from generalized convulsive SE, in which damage of limbic structures has been confirmed both pathologically and radiologically. It is thus unclear whether and when coma induction is indicated in forms of SE other than generalized convulsive SE. In fact, this approach could predispose to several complications (eg, pneumonia, deep vein thrombosis, pulmonary embolism, neuropathy, myopathy, ileus) [52].

We recently validated a clinical "Status Epilepticus Severity Score" (STESS) [38•, Class III], allowing an estimation of prognosis before treatment is instituted (Table 1). A favorable score has an excellent negative predictive value (0.97) for mortality: if the score is 0 to 2 , the patient will likely survive the SE episode. The score is extremely easy to use and may help to orient early treatment strategy in unclear situations. Patients having a favorable prediction may be managed less aggressively than those who have a potentially dismal prognosis, at least at the beginning. For patients with a favorable score, it appears advisable to try to avoid coma induction by administering nonsedating AEDs. If this approach fails to induce a notable clinical improvement after several hours (up to 24), elective coma induction may then be suggested.

SE episodes in patients with idiopathic generalized epilepsy (absence or myoclonic SE) readily respond to benzodiazepines and valproate; these patients should not be intubated. Conversely, postanoxic SE, the expression of a severe underlying encephalopathy, is often refractory to standard treatments, but in selected cases, after considering other prognostic factors such as brainstem reflexes and somatosensory evoked potentials, antiepileptic compounds (including anesthetics) could be prescribed [53•, Class IV].

In summary, every form of SE should always be treated quickly, but the depth of treatment should be tailored to the clinical situation. It is paramount to continue SE treatment, together with supportive care, in patients with episodes refractory to treatment for several weeks or months, as long as unmistakable evidence of irreversible neurologic damage is not found

\begin{tabular}{|c|c|c|}
\hline Criteria & Features & Score \\
\hline \multirow[t]{2}{*}{ Consciousness } & Alert or somnolent/confused & 0 \\
\hline & Stuporous or comatose & 1 \\
\hline \multirow[t]{3}{*}{ Worst seizure type } & Simple partial, complex partial, absence, myoclonic ${ }^{b}$ & 0 \\
\hline & Generalized convulsive & 1 \\
\hline & Nonconvulsive status epilepticus in coma & 2 \\
\hline \multirow[t]{2}{*}{ Age } & $<65$ y & 0 \\
\hline & $\geq 65 y$ & 2 \\
\hline \multirow[t]{2}{*}{ History of previous seizures } & Yes & 0 \\
\hline & No or unknown & 1 \\
\hline Total $^{\mathrm{a}}$ & & $0-6$ \\
\hline
\end{tabular}


[46,54; Class IV]. Once again, one has to keep in mind that etiology, age, and probably comorbidities are the most important predictors of outcome; the exact role of pharmacologic treatment, other than its supportive nature, has not yet been evaluated.

\section{Disclosure}

Dr. Rossetti has received speaker fees from UCB, has prepared scientific publications for Janssen-Cilag, and has received research support from Pfizer and UCB.

\section{References and Recommended Reading}

Papers of particular interest, published recently, have been highlighted as:

- Of importance,

$\bullet \quad$ Of major importance

1. Chen JW, Wasterlain CG: Status epilepticus: pathophysiology and management in adults. Lancet Neurol 2006, 5(3):246-256.

2. Lowenstein DH, Alldredge BK: Status epilepticus. $N$ Engl J Med 1998, 338(14):970-976.

3. Lowenstein DH, Bleck T, Macdonald RL: It's time to revise the definition of status epilepticus. Epilepsia 1999, 40(1):120-122.

4. Mazarati AM, Wasterlain CG: N-methyl-D-aspartate receptor antagonists abolish the maintenance phase of self-sustaining status epilepticus in rat. Neurosci Lett 1999, 265(3):187-190.

5. Holtkamp M, Othman J, Buchheim K, Meierkord H: Diagnosis of psychogenic nonepileptic status epilepticus in the emergency setting. Neurology 2006, 66(11):1727-1729.

6. Leppik IE, Derivan AT, Homan RW, et al.: Doubleblind study of lorazepam and diazepam in status epilepticus. JAMA 1983, 249(11):1452-1454.

7. Alldredge BK, Gelb AM, Isaacs SM, et al.: A comparison of lorazepam, diazepam, and placebo for the treatment of out-of-hospital status epilepticus. $N$ Engl J Med 2001, 345(9):631-637.

8. Treiman DM, Meyers PD, Walton NY, et al.: A comparison of four treatments for generalized convulsive status epilepticus. Veterans Affairs Status Epilepticus Cooperative Study Group. N Engl J Med 1998, 339 (12):792-798.

9. Treiman DM, Walton NY, Collins JF, VA Status Epilepticus Cooperative Study Group: Treatment of status epilepticus if first drug fails [abstract J.02]. Epilepsia 1999, 40(Suppl 7):243.

10. Treiman DM: Pharmacokinetics and clinical use of benzodiazepines in the management of status epilepticus. Epilepsia 1989, 30(Suppl 2):S4-S10.
11. Browne TR: The pharmacokinetics of agents used to treat status epilepticus. Neurology 1990, 40(5 Suppl 2):28-32.

12. Crevoisier C, Delisle MC, Joseph I, Foletti G: Comparative single-dose pharmacokinetics of clonazepam following intravenous, intramuscular and oral administration to healthy volunteers. Eur Neurol 2003, 49(3):173-177.

13. McIntyre J, Robertson S, Norris E, et al:: Safety and efficacy of buccal midazolam versus rectal diazepam for emergency treatment of seizures in children: a randomised controlled trial. Lancet 2005, 366 (9481):205-210.

14. Misra UK, Kalita J, Patel R: Sodium valproate vs phenytoin in status epilepticus: a pilot study. Neurology 2006, 67(2):340-342.

15. Sheth RD, Gidal BE: Intravenous valproic acid for myoclonic status epilepticus. Neurology 2000, 54 (5):1201.

16. Agarwal P, Kumar N, Chandra R, et al.: Randomized study of intravenous valproate and phenytoin in status epilepticus. Seizure 2007, 16(6):527-532.

17. Gilad R, Izkovitz N, Dabby R, et al.: Treatment of status epilepticus and acute repetitive seizures with i.v. valproic acid vs phenytoin. Acta Neurol Scand 2008, 118(5):296-300.

18. Rossetti AO, Bromfield EB: Determinants of success in the use of oral levetiracetam in status epilepticus. Epilepsy Behav 2006, 8(3):651-654.

19. Ruegg S, Naegelin Y, Hardmeier M, et al.: Intravenous levetiracetam: treatment experience with the first 50 critically ill patients. Epilepsy Behav 2008, 12(3):477480.

This is a detailed report on 50 patients who were treated with levetiracetam in the ICU. 
20. Knake S, Gruener J, Hattemer K, et al.: Intravenous levetiracetam in the treatment of benzodiazepine refractory status epilepticus. J Neurol Neurosurg Psychiatry 2008, 79(5):588-589.

The authors describe their experience with levetiracetam in the treatment of 18 patients with SE refractory to benzodiazepines

21. Kellinghaus C, Berning S, Besselmann M: Intravenous lacosamide as successful treatment for nonconvulsive status epilepticus after failure of first-line therapy. Epilepsy Behav 2009, 14(2):429-431.

This is the first report on the use of lacosamide in SE

22. Walton NY, Uthman BM, El Yafi K, et al.: Phenytoin penetration into brain after administration of phenytoin or fosphenytoin. Epilepsia 1999, 40 (2):153-156.

23. Yu KT, Mills S, Thompson N, Cunanan C: Safety and efficacy of intravenous valproate in pediatric status epilepticus and acute repetitive seizures. Epilepsia 2003, 44(5):724-726.

24. Perucca E: Pharmacological and therapeutic properties of valproate: a summary after 35 years of clinical experience. CNS Drugs 2002, 16(10):695-714.

25. Sinha $S$, Naritoku DK: Intravenous valproate is well tolerated in unstable patients with status epilepticus. Neurology 2000, 55(5):722-724.

26. Edwards KR, Glantz MJ: The pharmacokinetics of levetiracetam in human cerebrospinal fluid and serum: a controlled dose-ranging study in malignant brain-tumor patients. Epilepsia 2004, 45 (Suppl 7):121.

27. Fountain NB, Lothman EW: Pathophysiology of status epilepticus. J Clin Neurophysiol 1995, 12(4):326-342.

28. $\bullet$ Holtkamp M: The anaesthetic and intensive care of status epilepticus. Curr Opin Neurol 2007, 20 (2):188-193.

This article provides a complete and careful review of treatment options for refractory SE.

29. Claassen J, Hirsch LJ, Emerson RG, Mayer SA: Treatment of refractory status epilepticus with pentobarbital, propofol, or midazolam: a systematic review. Epilepsia 2002, 43(2):146-153.

30. Rossetti AO, Logroscino G, Bromfield EB: Refractory status epilepticus: effect of treatment aggressiveness on prognosis. Arch Neurol 2005, 62(11):16981702.

31. Bayliff CD, Schwartz ML, Hardy BG: Pharmacokinetics of high-dose pentobarbital in severe head trauma. Clin Pharmacol Ther 1985, 38(4):457-461.

32. Wessen A, Persson PM, Nilsson A, Hartvig P: Clinical pharmacokinetics of propofol given as a constantrate infusion and in combination with epidural blockade. J Clin Anesth 1994, 6(3):193-198.

33. Vasile B, Rasulo F, Candiani A, Latronico N: The pathophysiology of propofol infusion syndrome: a simple name for a complex syndrome. Intensive Care Med 2003, 29(9):1417-1425.

34. - Zarovnaya EL, Jobst BC, Harris BT: Propofolassociated fatal myocardial failure and rhabdomyolysis in an adult with status epilepticus. Epilepsia 2007, 48(5):1002-1006.

This is a detailed description of a patient developing a propofol infusion syndrome during SE treatment.

35. Rossetti AO, Reichhart MD, Schaller MD, et al.: Propofol treatment of refractory status epilepticus: a study of 31 episodes. Epilepsia 2004, 45(7):757-763.

36. Naritoku DK, Sinha S: Prolongation of midazolam half-life after sustained infusion for status epilepticus. Neurology 2000, 54(6):1366-1368.

37. Claassen J, Hirsch LJ, Emerson RG, et al.: Continuous EEG monitoring and midazolam infusion for refractory nonconvulsive status epilepticus. Neurology 2001 57(6):1036-1042.

38. Rossetti AO, Logroscino G, Milligan TA, et al.: Status Epilepticus Severity Score (STESS): a tool to orient early treatment strategy. J Neurol 2008, 255 (10):1561-1566.

This article reports on a multicenter validation study of the STESS score for the early estimation of prognosis in patients with SE.

39. Drislane FW, Blum AS, Lopez MR, et al.: Duration of refractory status epilepticus and outcome: loss of prognostic utility after several hours. Epilepsia 2009, 50(6):1566-1571

This retrospective analysis highlights the idea that etiology is the most important predictor of SE outcome.

40. Holtkamp M, Othman J, Buchheim K, et al.: A "malignant" variant of status epilepticus. Arch Neurol 2005, 62(9):1428-1431.

41. Pruss H, Holtkamp M: Ketamine successfully terminates malignant status epilepticus. Epilepsy Res 2008, 82(2-3):219-222.

This is a review of the literature and a description of one patient treated with ketamine for refractory SE.

42. Mirsattari SM, Sharpe MD, Young GB: Treatment of refractory status epilepticus with inhalational anesthetic agents isoflurane and desflurane. Arch Neurol 2004, 61(8):1254-1259.

43. Towne AR, Garnett LK, Waterhouse EJ, et al.: The use of topiramate in refractory status epilepticus. Neurology 2003, 60(2):332-334.

44. Iannetti P, Spalice A, Parisi P: Calcium-channel blocker verapamil administration in prolonged and refractory status epilepticus. Epilepsia 2005, 46 (6):967-969.

45. Bodenant M, Moreau C, Sejourne C, et al.: [Interest of the ketogenic diet in a refractory status epilepticus in adults]. Rev Neurol (Paris) 2008, 164(2):194-199.

46. Robakis TK, Hirsch LJ: Literature review, case report, and expert discussion of prolonged refractory status epilepticus. Neurocrit Care 2006, 4(1):35-46. 
47. De Herdt V, Waterschoot L, Vonck K, et al.: Vagus nerve stimulation for refractory status epilepticus. Eur J Paediatr Neurol 2009, 13(3):286-289.

48. Corry JJ, Dhar R, Murphy T, Diringer MN: Hypothermia for refractory status epilepticus. Neurocrit Care 2008, 9(2):189-197.

Exploratory report on four patients treated with mild hypothermia for refractory SE.

49. Lhatoo SD, Alexopoulos AV: The surgical treatment of status epilepticus. Epilepsia 2007, 48(Suppl 8):61-65.

50. Jordan KG, Hirsch LJ: In nonconvulsive status epilepticus (NCSE), treat to burst-suppression: pro and con. Epilepsia 2006, 47(Suppl 1):41-45.

51. Kaplan PW: No, some types of nonconvulsive status epilepticus cause little permanent neurologic sequelae (or: "the cure may be worse than the disease"). Neurophysiol Clin 2000, 30(6):377382.

52. Cereda C, Berger MM, Rossetti AO: Bowel ischemia: a rare complication of thiopental treatment for status epilepticus. Neurocrit Care 2009, 10(3):355358.

53. Rossetti AO, Oddo M, Liaudet L, Kaplan PW: Predictors of awakening from postanoxic status epilepticus after therapeutic hypothermia. Neurology 2009, 72(8):744-749.

This study identifies preserved EEG reactivity, brainstem reflexes, and somatosensory evoked potentials as favorable predictors of a response to treatment of postanoxic SE.

54. Dara SI, Tungpalan LA, Manno EM, et al.: Prolonged coma from refractory status epilepticus. Neurocrit Care 2006, 4(2):140-142. 